\title{
Identification of candidate target genes for endometrial cancer, such as ANO1, using weighted gene co-expression network analysis
}

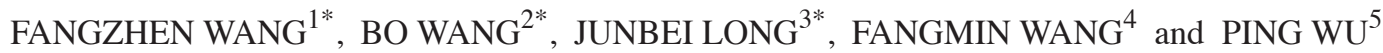 \\ ${ }^{1}$ The Outpatient Office, Affiliated Hospital of Xiangyang Vocational and Technical College, Xiangyang, Hubei 441000; \\ ${ }^{2}$ Department of Gynecology, Maternal and Child Health Hospital of Hubei Province, Wuhan, Hubei 430070; \\ ${ }^{3}$ Department of Radiology, Medical School of Xiangyang Vocational and Technical College, Xiangyang, Hubei 441000; \\ ${ }^{4}$ Department of Gynecology and Obstetrics, Zaoyang Third People's Hospital, Xiangyang, Hubei 441000; \\ ${ }^{5}$ Department of Oncology, Xiangyang Central Hospital, Xiangyang, Hubei 441000, P.R. China
}

Received May 14, 2018; Accepted September 14, 2018

DOI: $10.3892 /$ etm.2018.6965

\begin{abstract}
Network-based systems biology has become an important method for analysis of high-throughput gene expression data and gene function mining. The aim of the present study was to implement a weighted gene co-expression network analysis to screen genes that were significantly correlated with the clinical phenotype of endometrial cancer based on data from The Cancer Genome Atlas. By using the function 'pickSoftThreshold' in R software, the optimum soft thresholding power was determined to be 4 . Subsequently, a total of 2,414 expressed genes were identified among 19,791 genes from 506 samples, which were divided into 24 modules according to the different expression patterns. After analyzing the correlation between the gene expression in these 24 modules and the clinical phenotype of endometrial cancer, the anoctamin 1 (ANO1) gene was selected for further analysis. The Chi-squared test indicated that ANO1 was significantly associated with age $(\mathrm{P}=0.047)$, histological type $(\mathrm{P}<0.001)$, clinical stage $(\mathrm{P}<0.001)$, pathological grade $(\mathrm{P}<0.001)$ and positive peritoneal washing $(\mathrm{P}=0.001)$ of endometrial carcinoma. Kaplan-Meier survival analysis revealed that a high level of ANO1 was significantly associated with a good prognosis for endometrial cancer patients. Univariate and multivariate Cox regression analysis indicated that ANO1 is an independent prognostic factor in endometrial cancer. Further characterization of the most relevant module containing ANO1 with the database for annotation, visualization and integrated discovery tool suggested that ANO1 is involved in various pathways, including metabolic pathways. The present study suggests
\end{abstract}

Correspondence to: Ms. Bo Wang, Department of Gynecology, Maternal and Child Health Hospital of Hubei Province, 745 Wuluo Road, Wuhan, Hubei 430070, P.R. China

E-mail: m13657260086@163.com

*Contributed equally

Key words: endometrial cancer, The Cancer Genome Atlas, weighted gene correlation network analysis, anoctamin 1, overall survival that ANO1 may be a potential marker for good prognosis in endometrial cancer.

\section{Introduction}

Endometrial cancer was the fourth most common cancer in women, and $\sim 61,380$ new cases and $\sim 23,110$ associated deaths were registered in the USA in 2017 (1). While surgery has a high success rate for endometrial cancer at the early stage, the subset of patients with an advanced stage at diagnosis or with recurrent disease has poor treatment outcomes and low survival rates (2). Early detection of endometrial cancer may significantly improve the prognosis of affected patients. Therefore, the clinical implementation of screening for early-stage cancer prior to the onset of symptoms is expected to significantly improve the overall survival (OS). However, at present, effective methods for the early diagnosis of endometrial cancer are lacking and routine screening is not performed (3). A large quantity of studies have indicated that tumor markers may be used as indicators for the diagnosis of cancer and prognostication of affected patients, which may provide information that may be useful regarding their clinical management and follow-up (4).

The huge public transcriptome database provides a valuable resource for the analysis of whole-genome co-expression networks, screening of tumor markers associated with prognosis and phenotypes and investigation of the molecular mechanisms of pathogenesis (5). The weighted gene co-expression network analysis (WGCNA) method was developed by Langfelder and Horvath (6) in 2008. WGCNA has been proved to be an effective systems biology method to describe the correlation patterns among genes across microarray samples and to group genes into a model or network based on highly correlated expression profiles. Module eigengenes may be used to correlate modules with clinical traits to screen genes that may be used to identify candidate biomarkers or therapeutic targets. These methods have been successfully implemented in various biological contexts (6). In the present study, the WGCNA algorithm was applied to identify genes associated with clinical parameters of endometrial cancer, and 24 gene co-expression modules including 2,414 genes were retrieved, containing the anoctamin 1 (ANO1) gene (Fig. 1). 
ANO1, also known as transmembrane member 16A (TMEM16A), oral cancer overexpressed 2, DOG1, TAOS2, FLJ10261 or $\mathrm{Ca}^{2+}$-activated $\mathrm{Cl}^{-}$channel, is one of the 10 members of the transmembrane protein family (TMEM16A-K or ANO1-10) and was identified in 2008 (7). The ANO1 gene is localized on 11q13 (8), one of the most frequently amplified regions in human cancers. It has recently been indicated that ANO1/TMEM16A is amplified or overexpressed in several human cancer types and associated with a poor prognosis, including gastrointestinal stromal tumors $(9,10)$, pancreatic ductal adenocarcinoma (11), prostate cancer (12), head and neck squamous cell carcinoma (HNSCC) (13), breast cancer (14-16), lung cancer (17) and colorectal cancer (18). To date, only two studies in the pubmed database have examined the expression of ANO1 in endometrial cancer, one of which detected 10 endometrioid adenocarcinoma tissues by immunohistochemistry (IHC) with the new antibody K9, and only 4 of them were positive (19). The other study reported that no ANO1 expression was present in all 14 endometrial stromal sarcoma samples with the tyrosine 3-monooxygenase/tryptophan 5-monooxygenase activation protein epsilon (YWHAE)-NUT family member 2 (NUTM2) $\mathrm{A} / \mathrm{B}$ genetic fusion (20). However, the correlation between the expression of ANO1 in endometrial carcinoma and the clinical phenotype and prognosis have remained elusive. Therefore, in the present study, the expression of ANO1 was investigated and its clinical significance in endometrial cancer was determined.

\section{Materials and methods}

Gene expression profiles. mRNA expression data obtained through RNA-sequencing and clinical follow-up information from 547 endometrial cancer samples were downloaded from The Cancer Genome Atlas (TCGA) data portal (https:/tcga-data.nci.nih.gov/tcga/) in March 2018, including 19,791 human genes. A total of 41 patients were excluded due to missing information.

WGCNA. Screening of genes associated with the endometrial cancer phenotype was performed based on the constructed weighted correlation matrices according to the WGCNA protocols. The detailed steps are in accordance with those described by Langfelder and Horvath (6).

Functional enrichment analysis. The Kyoto Encyclopedia of Genes and Genomes (KEGG) is a tool for the systematic analysis of gene function, connecting genomic information with higher-order functional information. Gene Ontology (GO) anaylsis was performed to analyze ANO1 at the functional level, included biological processes, cellular component and molecular function. In the present study, GO and KEGG pathway analysis was performed using the database for annotation, visualization and integrated discovery (DAVID) platform (https://david. ncifcrf.gov/).

Statistical analysis. The Chi-square test was performed to evaluate the association between the RNA expression of ANO1 and clinical characteristics. Survival analysis was performed using the Kaplan-Meier method and significant differences between subgroups were determined using the log-rank test. Univariate/multivariate Cox proportional hazard regression

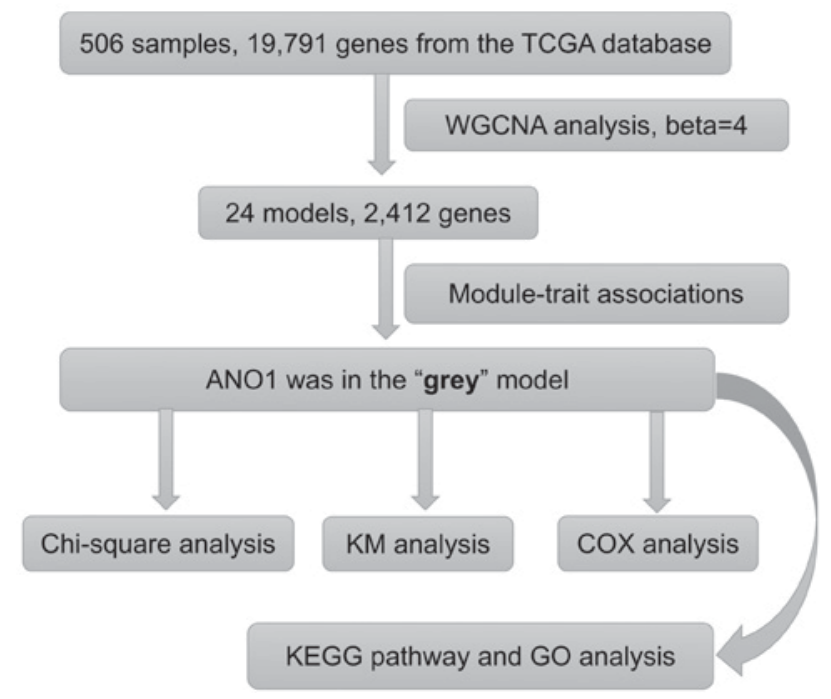

Figure 1. Schematic illustrating the sequence of data analyses to screen genes associated with the clinical phenotype of endometrial cancer. TCGA, The Cancer Genome Atlas; KM, Kaplan-Meier; KEGG, Kyoto Encyclopedia of Genes and Genomes; GO gene ontology; WGCNA, weighted gene correlation network analysis; ANO1, anoctamin 1.

analysis was performed to identify factors influencing survival. The cut-off values for the genes were determined using X-tile software (version 3.6.1) (21). $\mathrm{P}<0.05$ was considered to indicate a statistically significant difference. Statistical analyses were performed using SPSS software version 22.0 (IBM Corp., Armonk, NY, USA). WGCNA was performed using R software version 3.4.1 (https://www.r-project.org/). The 'WGCNA' package was downloaded from https://mran.microsoft. com/package/WGCNA.

\section{Results}

Patient characteristics. All of the 506 patients included in the present study were clinically and pathologically diagnosed with endometrial cancer between 1995 and 2013. The mean age of these patients was 64 years (range, 31-90 years). According to the International Federation of Gynaecology and Obstetrics staging guidelines from 2009 (22), the cohort included 310 patients $(61.26 \%)$ with stage I, 48 (9.49\%) with stage II, 121 (23.91\%) with stage III and $27(5.35 \%)$ with stage IV endometrial cancer. Among the 506 cases, 376 (74.31\%) were histologically classified as endometrioid endometrial adenocarcinoma, $22(4.35 \%)$ were mixed serous and endometrioid adenocarcinoma and 108 (21.34\%) were serous endometrial adenocarcinoma. The distribution of histological grades was as follows: A total of 104 cases $(20.55 \%)$ were of grade 1, $110(21.74 \%)$ were of grade 2 and $292(57.71 \%)$ were of grade 3 .

Screening of genes associated with the phenotype of endometrial carcinoma. To identify genes that may be significantly associated with the endometrial carcinoma phenotype, a WGCNA was employed to identify modules of highly interconnected genes. A gene co-expression network was constructed on the basis of pairwise correlations of the expression of highly interconnected genes with a significantly 
A
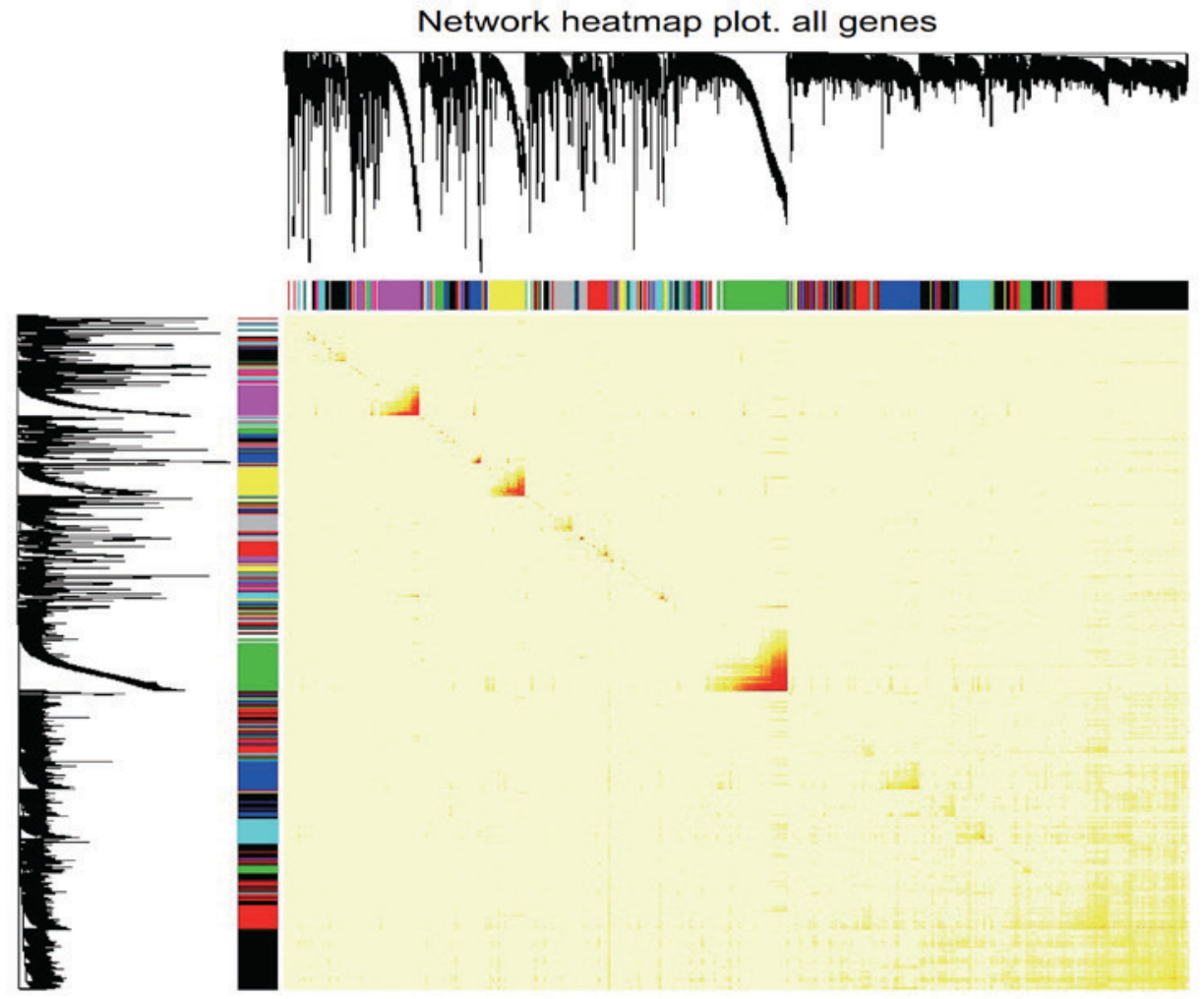

|||||||||| || |||| |||| | | |||||||||||||

B

Scale independence

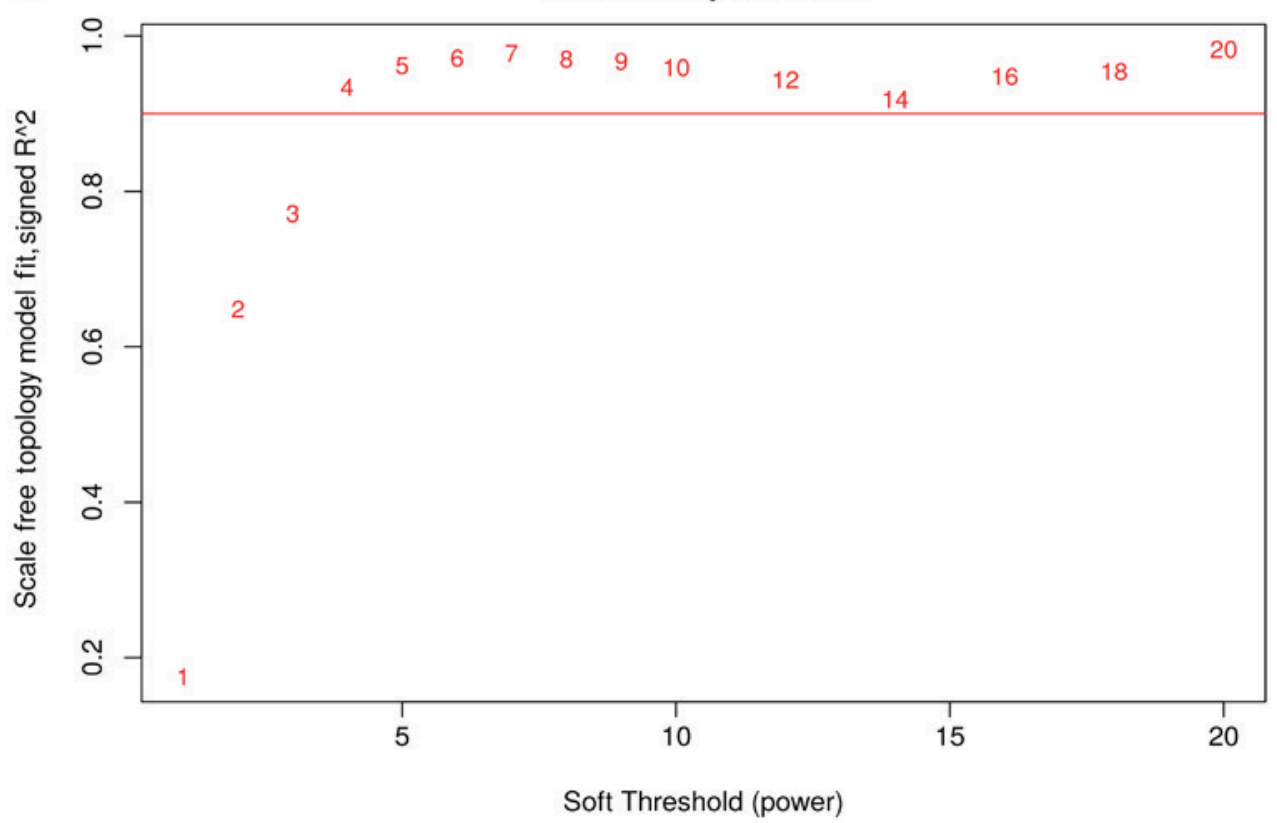

Figure 2. (A) Construction of a whole genome co-expression network. Different color bands representing different modules. Each point in the map indicates a strong (red) or weak (yellow) correlation between genes. (B) Analysis of the scale-free fit index for soft threshold powers ( $\beta$ ). In the presented study, the power of $\beta=4$ (the red line: Scale free R2=0.95) was selected as the soft-thresholding parameter to ensure a scale-free network.

correlated co-expression by using the total of 19,791 genes (Fig. 2A). Though the function 'PickSoftThreshold' in R software, the optimum soft thresholding power was calculated as 4 (Fig. 2B). Subsequently, a total of 2,414 expressed genes were screened from 19,791 genes in 506 samples, which were divided into 24 modules according to the different expression patterns via average linkage hierarchical clustering (Fig. 3A and B). The association between seven clinical phenotypes (histological type, clinical stage, pathological grade, positive peritoneal washing, ethnicity, residual tumor and menopause status) and genome-wide expression profiles were investigated using the tool 'Eigengene Networks' (Fig. 4). As depicted in Fig. 4, the 'grey' module was associated with each clinical phenotype and was most highly correlated with the histological type (coefficient $r=0.72$; $\mathrm{P}<0.001)$. By consulting the literature, ANO1 was selected 
A

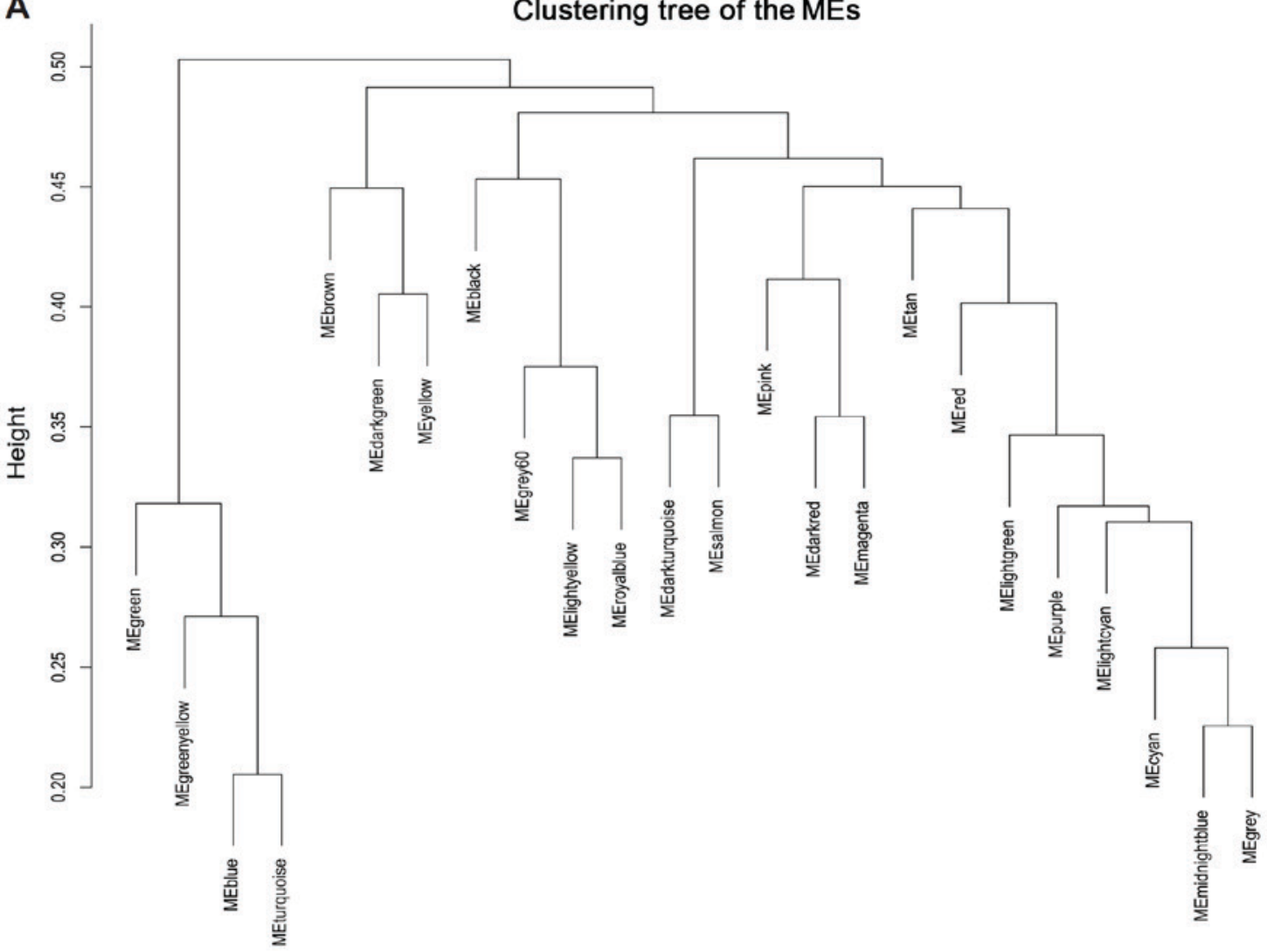

B

Gene dendrogram and module colors

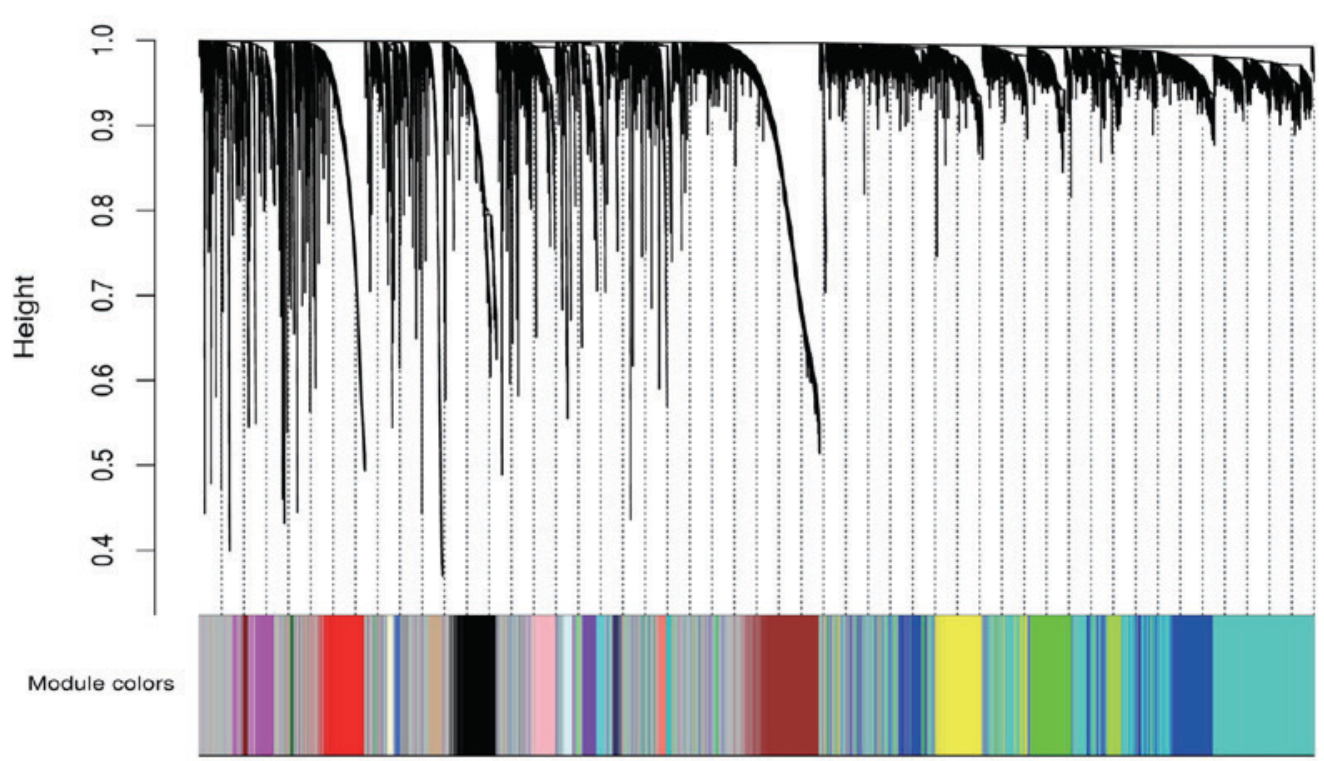

Figure 3. Dendrogram of all differentially expressed genes clustered based on a dissimilarity measure. The modules with a soft threshold power of $>4$ were selected. (A) Clustering tree of the MEs; (B) clustering dendrogram of genes. The dissimilarity of genes was based on topological overlap. The genes were assigned to different modules and were identified using different colors. ME, module eigengene.

for further study as it as determined to be involved in endometrial cancer (7-20). The ANO1 gene was in the 'grey' module and closely correlated with the clinical phenotype of the endometrial cancer patients (Figs. 1 and 5).
Clinicopathological characteristics. The results of the Chi-square analysis further confirmed the prediction results of WCGNA, as ANO1 mRNA expression was significantly associated with age $(\mathrm{P}=0.047)$, histological type $(\mathrm{P}<0.001)$, clinical 
Module-trait associations

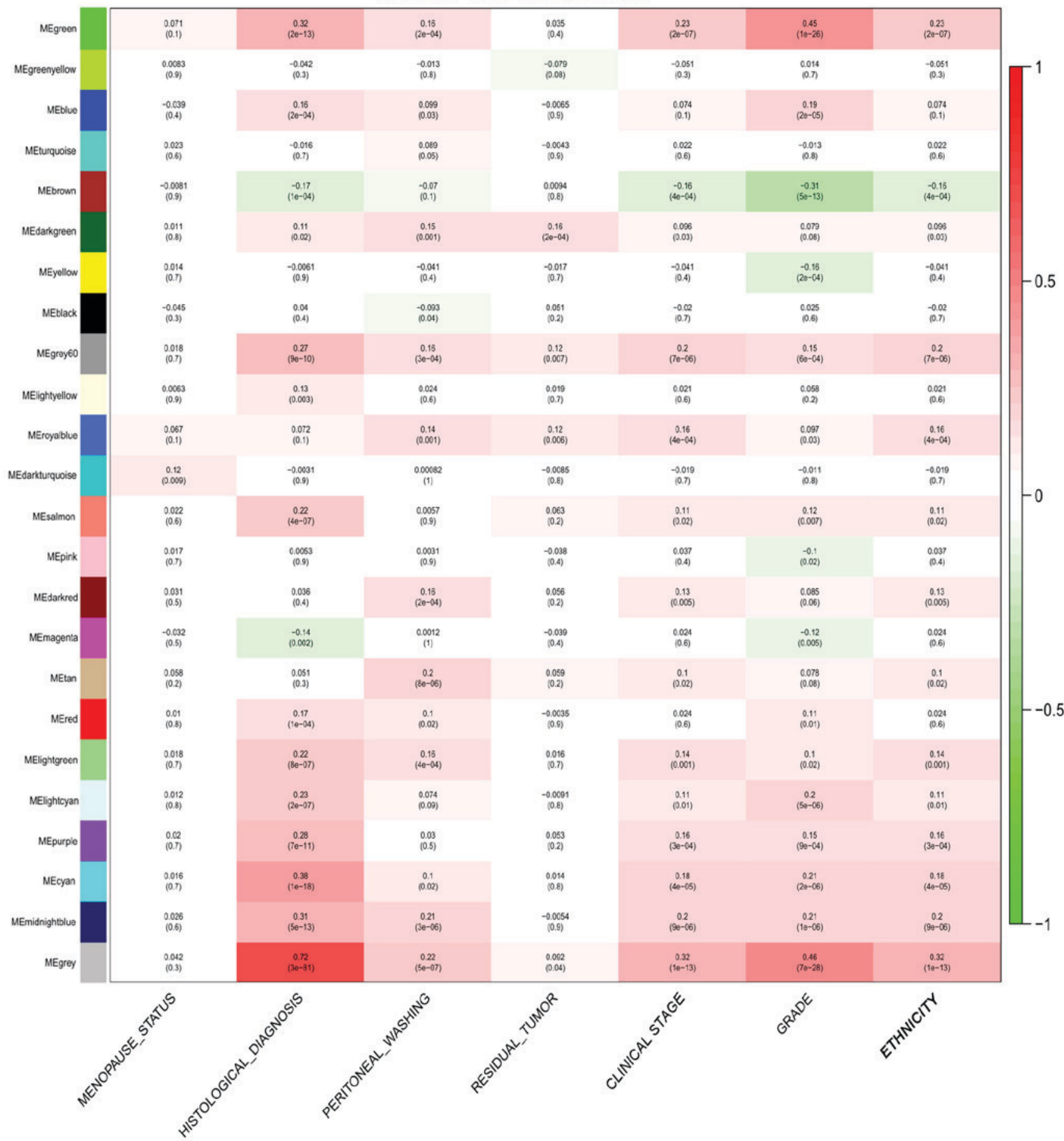

Figure 4. Associations of consensus module eigengenes and the clinical phenotype of endometrial cancer. The module name is displayed in left-hand panel. Numbers in the table indicate the correlations of the corresponding module eigengenes and clinical phenotype, with P-values displayed below the correlations in brackets. The intensity and direction of associations are indicated on the right side of the heatmap (red, positively correlated; green, negatively correlated). ME, module eigengene.

stage $(\mathrm{P}<0.001)$, pathological grade $(\mathrm{P}<0.001)$ and positive peritoneal washing $(\mathrm{P}<0.001)$ of endometrial carcinoma. However, no correlation with the patients' menopause status was identified ( $\mathrm{P}=0.893$; Table I).

Prognostic significance. To estimate the clinical prognostic significance of ANO1 mRNA expression, a Kaplan-Meier survival analysis was performed. The cut-off value $(0.5)$ of the relative mRNA expression of ANO1 was computed by X-tile; patietns with endometrial cancer were stratified into lowand high-risk groups. The number of subjects in the ANO1 high-risk group and low-risk group was 273 and 231, respectively. As presented in Fig. 6, patients with a lower expression of ANO1 had a worse prognosis $(\mathrm{P}<0.001)$. To assess whether the prognostic power of ANO1 was independent of other clinical features, univariate and multivariable Cox regression analyses were performed, the results demonstrated that the prognostic power of ANO1 for the prediction of survival rate 
Table I. Association of ANO1 with clinicopathological characteristics in endometrial cancer patients $(n=506)$.

Risk based on ANO1 expression ${ }^{\mathrm{a}}$

\begin{tabular}{|c|c|c|c|}
\hline Parameter & High $(n=233)$ & Low $(n=273)$ & P-value \\
\hline Age (years) & & & 0.047 \\
\hline$\leq 64$ & $112(41.8)$ & $156(58.2)$ & \\
\hline$>64$ & $119(50.6)$ & $116(49.4)$ & \\
\hline Not available & $2(66.7)$ & $1(33.3 \%)$ & \\
\hline Histological type & & & $<0.001$ \\
\hline Endometrioid endometrial adenocarcinoma & $134(35.6)$ & $242(64.4)$ & \\
\hline Mixed serous and endometrioid & $11(50.0)$ & $11(50.0)$ & \\
\hline Serous endometrial adenocarcinoma & $88(81.5)$ & $20(18.5)$ & \\
\hline Grade & & & $<0.001$ \\
\hline G1 & $25(24.0)$ & $79(76.0)$ & \\
\hline G2 & $30(27.3)$ & $80(72.7)$ & \\
\hline G3 & $178(61.0)$ & $114(39.0)$ & \\
\hline FIGO stage & & & $<0.001$ \\
\hline I & $116(37.4)$ & $194(62.6)$ & \\
\hline II & $20(41.7)$ & $28(58.3)$ & \\
\hline III & $74(61.2)$ & $47(38.8)$ & \\
\hline IV & $23(85.2)$ & $4(14.8)$ & \\
\hline Peritoneal washing & & & 0.001 \\
\hline Negative & $138(42.7)$ & $185(57.3)$ & \\
\hline Positive & $38(66.7)$ & $19(33.3)$ & \\
\hline Not available & $57(45.2 \%)$ & $69(54.8)$ & \\
\hline Menopausal status & & & 0.893 \\
\hline Period & $7(43.8)$ & $9(56.3)$ & \\
\hline Post & $192(46.4)$ & 222 (53.6) & \\
\hline Pre & $14(42.4)$ & $19(57.6)$ & \\
\hline Not available & $29(67.4)$ & $14(32.6)$ & \\
\hline
\end{tabular}

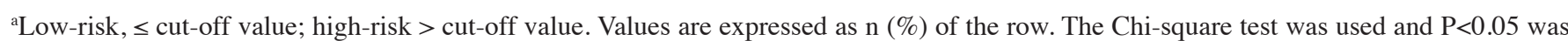
considered to indicate statistical significance. FIGO, International Federation of Gynaecology and Obstetrics; ANO1, anoctamin 1; LMP, last menstrual period; Period, 6-12 months since LMP; Post, prior bilateral ovariectomy or >12 months since LMP with no prior hysterectomy; Pre, $<6$ months since LMP, no prior bilateral ovariectomy and not on estrogen replacement.

was independent of these clinical features (age, histological type, clinical stage and pathological grade) for patients with endometrial cancer (High-risk group vs. Low-risk group, $\mathrm{HR}=1.904, \mathrm{P}=0.011$; Table II).

Functional analysis. To gain a primary understanding of the biological relevance of ANO1, KEGG pathway and GO enrichment analyses of the differentially expressed genes determined from the data from TCGA database were performed. Further characterization of the most relevant module containing the ANO1 gene with the DAVID tool indicated that genes co-expressed with ANO1 were mainly enriched in metabolic pathways (Fig. 7A). In Fig. 7B, significantly enriched GO terms in the categories biological process, cellular component and molecular function are listed. In the category biological process, genes co-expressed with ANO1 were mainly enriched in the term 'homophilic cell adhesion via plasma membrane adhesion molecule'. In the category cellular component, genes co-expressed with ANO1 were mainly enriched in the term 'integral component of membrane'. In the category molecular function, genes co-expressed with ANO1 were mainly enriched in the term 'bitter taste receptor activity' and also enriched in 'calcium ion binding'.

\section{Discussion}

The purpose of the present study was to screen out the factors associated with the endometrial carcinoma phenotype by WCGAN analysis using the resources in TCGA public database and to further verify whether they were associated with the phenotype and progression of endometrial cancer by statistical methods. Although several genes were identified following the literature review (7-20), ANO1 was selected for further analysis. The ANO1 gene was identified as an independent prognostic factor, providing a basis for further experimental verification using tissue and cell tests in the laboratory. 


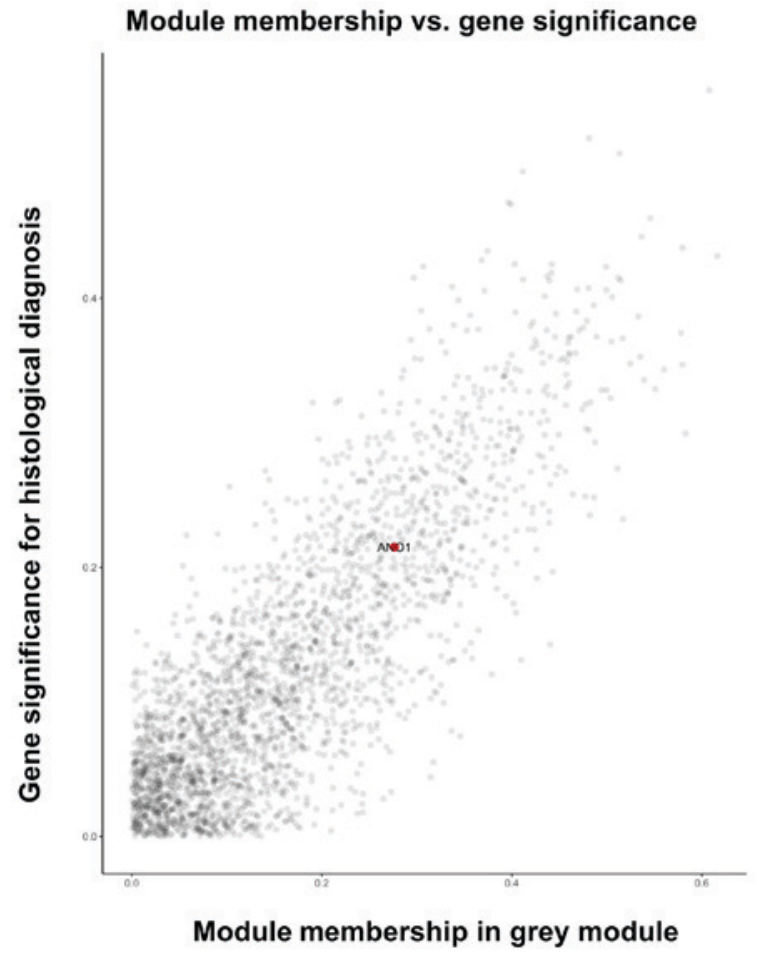

Figure 5. Module membership in the grey module. The ANO1 gene was included in the 'grey hub genes' module. ANO1, anoctamin 1.

The present analysis of TCGA database by WCGNA indicated that 2,414 genes, including ANO1, were associated with endometrial cancer; these were stratified into 24 subgroups. Subsequently, the Chi-square analysis revealed that the expression status of ANO1 was significantly associated with patient age, histological type, clinical stage, pathological grade and positive peritoneal washing of endometrial carcinoma. High ANO1 levels were significantly correlated with a good prognosis of endometrial cancer patients. Wu et al (15) assessed the expression of ANO1 in 431 breast cancer patients with invasive ductal carcinoma and identified that ANO1 overexpression was associated with good prognosis in progesterone receptor-positive or human epidermal growth factor receptor 2-negative patients following tamoxifen treatment. However, ANO1 has previously been reported to be overexpressed in numerous types of cancer and to be associated with poor prognosis. Bae et al (16) investigated the expression of ANO1 in 139 breast cancer patients and identified that ANO1 was significantly associated with poor prognosis in terms of a shorted OS and relapse-free survival rate of breast cancer patients. In gastrointestinal stromal tumors with mutations in KIT or PDGFRA, for which ANO1 had been previously indicated to be a specific biomarker, ANO1 was reported to be highly associated with tumor size and to be of predictive value (10). Jia et al (17) demonstrated that ANO1 overexpression contributes to tumor growth and invasion of lung cancer. In esophageal squamous cell carcinoma, Shi et al (23) identified that ANO1 mRNA expression was elevated in samples with moderate dysplasia compared with that in normal esophageal epithelium; ANO1 was overexpressed in esophageal squamous cell carcinoma, and positively correlated with lymph node metastasis and advanced clinical stage determined by IHC. By analyzing gnomic amplification and protein expression

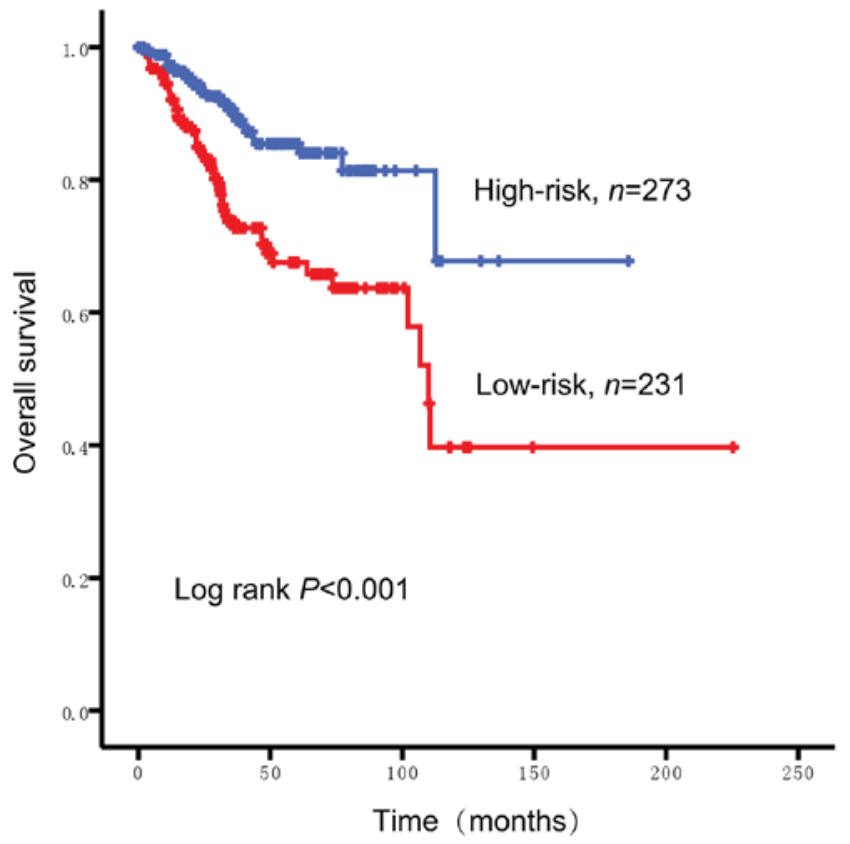

Figure 6. Kaplan-Meier curve analysis of the association of ANO1 expression with OS of endometrial cancer patients. The log-rank test indicated that the expression status of ANO1 was significantly associated with OS. The cut-off value of relative ANO1 expression was 0.5 . Low-risk, $\leq$ cut-off value of ANO1 expression; high-risk > cut-off value of ANO1 expression. ANO1, anoctamin 1; OS, overall survival.

of ANO1 in a large number of HNSCC patients, Ruiz et al (13) revealed that ANO1 protein expression in cancer tissue was associated with poor OS. The abovementioned studies indicated that the role of the ANO1 gene may vary among different types of cancer.

The ANO1 gene was first discovered in gastric stromal cancer and was initially named DOG1 (24). ANO1, as an ion channel, has a crucial role in sensing and transmitting extracellular signals to the intracellular machinery of epithelial cells, and a dysfunction of ANO1 may be involved in the development and progression of cancer of the epithelium by causing alterations of ion homeostasis and volume regulation $(25,26)$. The ANO1 gene is involved in the development of cancer based on regulating intracellular free $\mathrm{Ca}^{2+}$ levels $(12,27)$. In the present study, GO functional enrichment and KEGG pathway analysis indicated that genes co-expressed with ANO1 were mainly enriched in the term 'homophilic cell adhesion via plasma membrane adhesion molecule' and involved in the $\mathrm{Ca}^{2+}$ ion metabolism pathway. This result in the current study was consistent with the existing literature.

The expression of ANO1 in endometrial cancer has been assessed by only two previous studies. One of the studies examined 10 endometrioid adenocarcinoma tissues with the new antibody K9 and the results indicated that only 4 of them were positive (19). The other study suggested that ANO1 was negatively expressed in all 14 endometrial stromal sarcomas with the YWHAE-NUTM2A/B genetic fusion (20). The Human Protein Atlas database (https://www.proteinatlas.org/search/ANO1) (28) contains data obtained by IHC analysis of 11 endometrial carcinoma samples using an anti-ANO1 antibody (cat. no. HPA032148; Sigma-Aldrich; Merck KGaA). The results indicated high expression in 2 cases, very high expression in 6 cases and weak 
Table II. Univariate and multivariate Cox regression analysis in endometrial cancer patients regarding overall survival.

\begin{tabular}{|c|c|c|c|c|}
\hline \multirow[b]{2}{*}{ Characteristic } & \multicolumn{2}{|c|}{ Univariate analysis } & \multicolumn{2}{|c|}{ Multivariate analysis } \\
\hline & HR $(95 \% \mathrm{CI})$ & P-value & $\mathrm{HR}(95 \% \mathrm{CI})$ & P-value \\
\hline Age ( $\leq 64$ vs. $>64$ years) & $1.568(1.018-2.416)$ & 0.041 & $1.738(1.103-2.738)$ & 0.017 \\
\hline Histological type (EEA vs. SEA) & $2.785(1.772-4.377)$ & $<0.001$ & & 0.223 \\
\hline Grade (G1 vs. G2+G3) & $3.002(1.448-6.225)$ & 0.003 & & 0.90 \\
\hline FIGO stage (I/II vs. III/IV) & $4.026(2.605-6.223)$ & $<0.001$ & $0.297(0.186-0.476)$ & $<0.001$ \\
\hline Risk based on ANO1 (high vs. low) ${ }^{\mathrm{a}}$ & $0.397(0.252-0.626)$ & $<0.001$ & $1.904(1.158-3.130)$ & 0.011 \\
\hline
\end{tabular}

The cut-off value of relative ANO1 expression was 0.5. ${ }^{a}$ Low-risk, $\leq$ cut-off value; high-risk, $>$ cut-off value. EEA, endometrioid endometrial adenocarcinoma; SEA, serous endometrial adenocarcinoma; HR, hazard ratio; FIGO, International Federation of Gynaecology and Obstetrics; CI, confidence interval; ANO1, anoctamin 1.

A

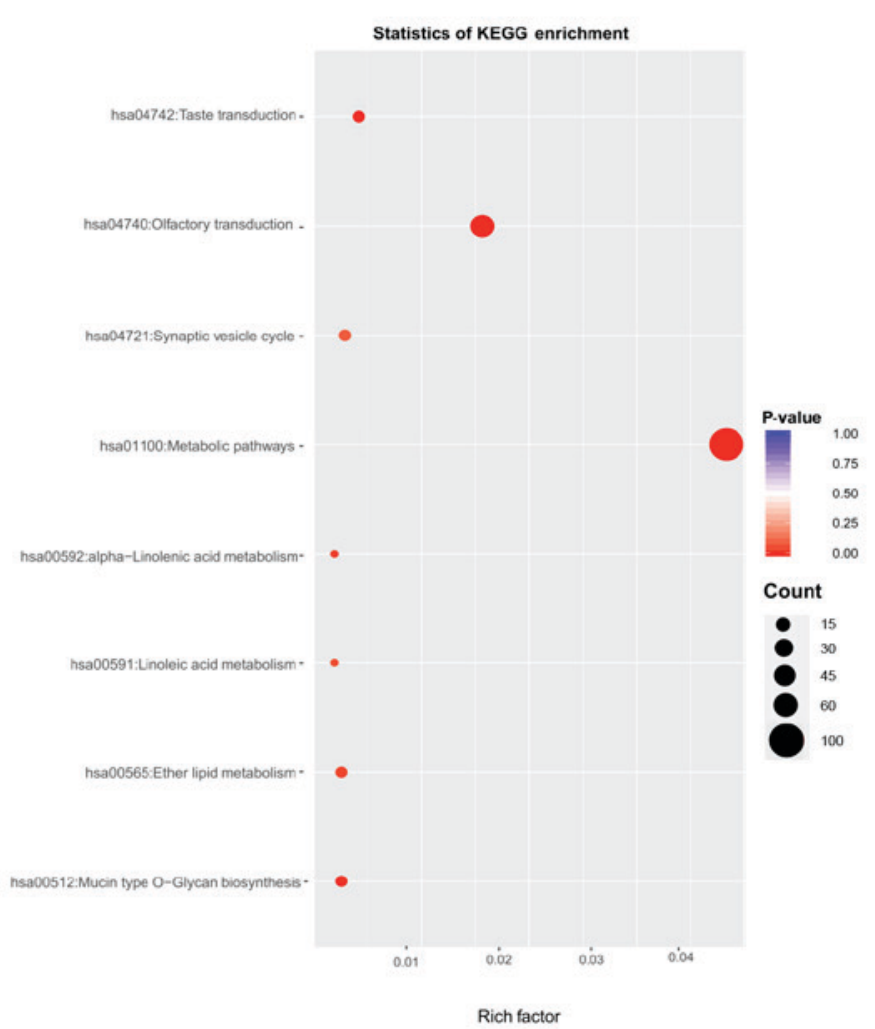

B

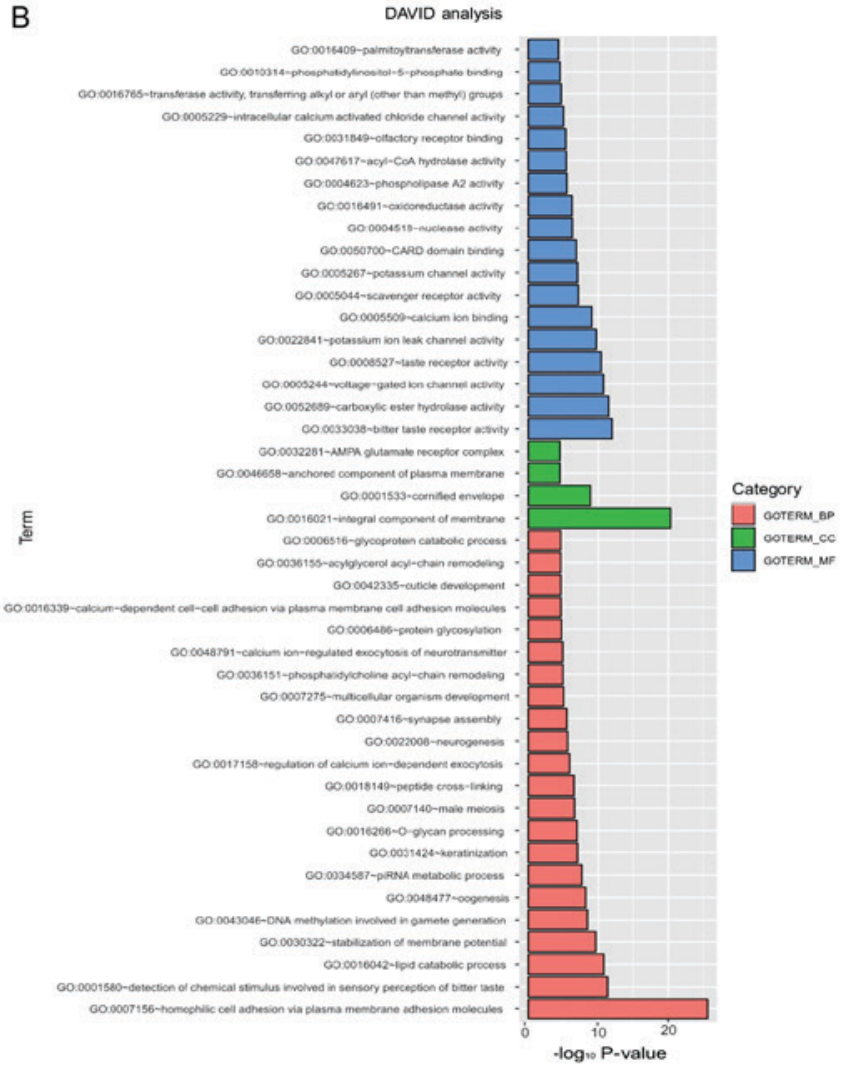

Figure 7. Functional analysis. (A) The significantly enriched KEGG pathways of the genes co-expressed with ANO1; (B) significantly enriched GO terms in the categories BP, CC and MF for the genes co-expressed with ANO1. ANO1, anoctamin 1; KEGG, Kyoto Encyclopedia of Genes and Genomes; hsa, Homo sapiens; GO, Gene Ontology; DAVID, database for annotation, visualization and integrated discovery; BP, Biological Process; CC, Cellular Component; MF, Molecular Function.

expression in 3 cases, and the intensity of ANO1 expression was different in endometrial cancer tissues of different stages. To the best of our knowledge, the correlation between ANO1 and the clinical phenotype and prognosis of endometrial carcinoma has not been previously assessed. In the present study, the expression of ANO1 was determined from data of endometrial cancer tissues downloaded from TCGA and its clinical significance was assessed. In addition, the number of subjects included in the two previous studies was relatively small; one comprised 10 endometrioid adenocarcinoma tissues and the other 14 endometrial stromal sarcomas $(19,20)$. The present study included a relatively large number of subjects, namely 506 patients with endometrial cancer. Based on the public data from TCGA database, factors associated with the clinical characteristics of endometrial cancer were screened out, and ANO1 was further investigated. It was revealed that high expression of ANO1 was associated with a good prognosis of endometrial carcinoma patients. This result provides a theoretical basis for further verifying the role and the potential prognostic value of ANO1 in endometrial cancer in laboratory studies to be performed in the future. These may 
include polymerase chain reaction and IHC analyses of a large number of cancer samples.

\section{Acknowledgements}

Not applicable.

\section{Funding}

No funding was received.

\section{Availability of data and materials}

The datasets analyzed during the current study are available in the TCGA repository, https://tcga-data.nci.nih.gov/tcga/; DAVID repository https://david.ncifcrf.gov/; R repository, https://www.r-project.org/ and the Human Prtotein Atlas repository, https://www.proteinatlas.org/search/ANO1.

\section{Authors' contributions}

Data curation: FzW, BW and JL; formal analysis: FmW and PW; drafting of the manuscript: $\mathrm{BW}$; critical review and editing of the manuscript: FzW and JL.

\section{Ethical approval and consent to participate}

Not applicable.

\section{Patient consent for publication}

Not applicable.

\section{Competing interests}

The authors declare that they have no competing of interests.

\section{References}

1. Siegel RL, Miller KD and Jemal A: Cancer statistics, 2017. CA Cancer J Clin 67: 7-30, 2017.

2. Amant F, Moerman P, Neven P, Timmerman D, Van Limbergen E and Vergote I: Endometrial cancer. Lancet 366: 491-505, 2005.

3. Berek JS: Novak's Gynecology 13th edition. Lippincott Williams \& Wilkins, Philadelphia, PA, USA, 2002.

4. Ueda Y, Enomoto T, Kimura T, Miyatake T, Yoshino K, Fujita M and Kimura T: Serum biomarkers for early detection of gynecologic cancers. Cancers (Basel) 2: 1312-1327, 2010.

5. Roychowdhury $\mathrm{S}$ and Chinnaiyan AM: Translating cancer genomes and transcriptomes for precision oncology. CA Cancer J Clin 66 $75-88,2015$.

6. Langfelder P and Horvath S: WGCNA: An R package for weighted correlation network analysis. BMC Bioinformatics 9: 559, 2008.

7. Caputo A, Caci E, Ferrera L, Pedemonte N, Barsanti C, Sondo E, Pfeffer U, Ravazzolo R, Zegarra-Moran O and Galietta LJ TMEM16A, a membrane protein associated with calcium-dependent chloride channel activity. Science 322: 590-594, 2008.

8. Katoh M and Katoh M: FLJ10261 gene, located within the CCND1-EMS1 locus on human chromosome 11q13, encodes the eight-transmembrane protein homologous to C12orf3, C11orf25 and FLJ34272 gene products. Int J Oncol 22: 1375-1381, 2003.

9. Berglund E, Akcakaya P, Berglund D, Karlsson F, Vukojevic V, Lee L, Bogdanović D, Lui WO, Larsson C, Zedenius J, et al: Functional role of the $\mathrm{Ca}^{2+}$-activated $\mathrm{Cl}^{-}$channel DOG1/TMEM16A in gastrointestinal stromal tumor cells. Exp Cell Res 326: 315-325, 2014.
10. Rizzo FM, Palmirotta R, Marzullo A, Resta N, Cives M, Tucci M and Silvestris F: Parallelism of DOG1 expression with recurrence risk in gastrointestinal stromal tumors bearing KIT or PDGFRA mutations. BMC Cancer 16: 87, 2016.

11. Sauter DR, Novak I, Pedersen SF, Larsen EH and Hoffmann EK: ANO1 (TMEM16A) in pancreatic ductal adenocarcinoma (PDAC). Pflugers Arch 467: 1495-1508, 2015.

12. Liu W, Lu M, Liu B, Huang Y and Wang K: Inhibition of $\mathrm{Ca}(2+)$-activated $\mathrm{Cl}(-)$ channel ANO1/TMEM16A expression suppresses tumor growth and invasiveness in human prostate carcinoma. Cancer Lett 326: 41-51, 2012.

13. Ruiz C, Martins JR, Rudin F, Schneider S, Dietsche T, Fischer CA, Tornillo L, Terracciano LM, Schreiber R, Bubendorf L and Kunzelmann K: Enhanced expression of ANO1 in head and neck squamous cell carcinoma causes cell migration and correlates with poor prognosis. PLoS One 7: e43265, 2012.

14. Ubby I, Bussani E, Colonna A, Stacul G, Locatelli M, Scudieri P, Galietta L and Pagani F: TMEM16A alternative splicing coordination in breast cancer. Mol Cancer 12: 75, 2013.

15. Wu H, Guan S, Sun M, Yu Z, Zhao L, He M, Zhao H, Yao W Wang E, Jin F, et al: Ano1/TMEM16A overexpression is associated with good prognosis in PR-positive or HER2-negative breast cancer patients following tamoxifen treatment. PLoS One 10: e0126128, 2015.

16. Bae JS, Park JY, Park SH, Ha SH, An AR, Noh SJ, Kwon KS, Jung SH, Park HS, Kang MJ and Jang KY: Expression of ANO1/DOG1 is associated with shorter survival and progression of breast carcinomas. Oncotarget 9: 607-621, 2017.

17. Jia L, Liu W, Guan L, Lu M and Wang K: Inhibition of calcium-activated chloride channel ANO1/TMEM16A suppresses tumor growth and invasion in human lung cancer. PLoS One 10: e 0136584,2015

18. Sui Y, Sun M, Wu F, Yang L, Di W, Zhang G, Zhong L, Ma Z, Zheng J, Fang $X$ and Ma T: Inhibition of TMEM16A expression suppresses growth and invasion in human colorectal cancer cells. PLoS One 9: e115443, 2014.

19. Hemminger J and Iwenofu OH: Discovered on gastrointestinal stromal tumours 1 (DOG1) expression in non-gastrointestinal stromal tumour (GIST) neoplasms. Histopathology 61: 170-177, 2012.

20. Lee CH, Hoang LN, Yip S, Reyes C, Marino-Enriquez A, Eilers G, Tao D, Chiang S, Fletcher JA, Soslow RA, et al: Frequent expression of KIT in endometrial stromal sarcoma with YWHAE genetic rearrangement. Mod Pathol 27: 751-757, 2014.

21. Camp RL, Dolled-Filhart M and Rimm DL: X-tile: A new bio-informatics tool for biomarker assessment and outcome-based cut-point optimization. Clin Cancer Res 10: 7252-7259, 2004.

22. Creasman W: Revised FIGO staging for carcinoma of the endometrium. Int J Gynaecol Obstet 105: 103, 2009.

23. Shi ZZ, Shang L, Jiang YY, Hao JJ, Zhang Y, Zhang TT, Lin DC, Liu SG, Wang BS, Gong T, et al: Consistent and differential genetic aberrations between esophageal dysplasia and squamous cell carcinoma detected by array comparative genomic hybridization. Clin Cancer Res 19: 5867-5878, 2013

24. Robert B, Corless CL, Chen X, Rubin BP, Subramanian S, Montgomery K, Zhu S, Ball CA, Nielsen TO, Patel R, et al: The novel marker, DOG1, is expressed ubiquitously in gastrointestinal stromal tumors irrespective of KIT or PDGFRA mutation status. Am J Pathol 165: 107-113, 2004.

25. Xu B, Jin X, Min L, Li Q, Deng L, Wu H, Lin G, Chen L, Zhang H, Li C, et al: Chloride channel-3 promotes tumor metastasis by regulating membrane ruffling and is associated with poor survival. Oncotarget 6: 2434-2450, 2015.

26. Kim TH, Kim JS, Kim ZH, Huang RB, Chae YL and Wang RS: Khz (Fusion Product of Ganoderma lucidum and Polyporus umbellatus Mycelia) induces apoptosis in human colon carcinoma HCT116 cells, accompanied by an increase in reactive oxygen species, activation of caspase 3 , and increased intracellular $\mathrm{Ca}^{2+}$. J Med Food 18: 332-336, 2015.

27. Mazzone A, Eisenman ST, Strege PR, Yao Z, Ordog T, Gibbons SJ and Farrugia G: Inhibition of cell proliferation by a selective inhibitor of the $\mathrm{Ca} 2(+)$-activated $\mathrm{Cl}(-)$ channel, Ano1. Biochem Biophys Res Commun 427: 248-253, 2012.

28. Pontén F, Jirström K and Uhlen M: The human protein Atlas-a tool for pathology. J Pathol 216: 387-393, 2008.

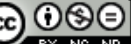

This work is licensed under a Creative Commons Attribution-NonCommercial-NoDerivatives 4.0 International (CC BY-NC-ND 4.0) License. 